\title{
Human Papillomavirus, p16, and Epidermal Growth Factor Receptor Biomarkers and CT Perfusion Values in Head and Neck Squamous Cell Carcinoma
}

N.L. Hoefling, J.B. McHugh, E. Light, B. Kumar, H. Walline, M. Prince, C. Bradford, T.E. Carey, and S.K. Mukherji

\begin{abstract}
BACKGROUND AND PURPOSE: Head and neck squamous cell carcinoma tumors positive for laboratory biomarkers hrHPV and p16 and negative for EGFR often respond better to nonsurgical organ-preservation therapy than hrHPV-negative, pl6-negative, and EGFR overexpressing tumors. CTP has been shown to distinguish which locally advanced head and neck squamous cell carcinomas will respond to induction chemotherapy or chemoradiation. Our purpose was to determine whether a relationship exists between CTP measures and the expression of these laboratory biomarkers, because both appear to separate head and neck squamous cell carcinoma tumors into similar groups.
\end{abstract}

MATERIALS AND METHODS: We conducted an institutional review board-approved, Health Insurance Portability and Accountability Act-compliant retrospective review of head and neck CTP in 25 patients with locally advanced head and neck squamous cell carcinoma who had signed informed consent. Eight women and 17 men, 41-80 years of age, constituted a pretreatment group of 18 patients and a palliative group of 7 patients. Tumor biopsy samples were analyzed for overexpression of hrHPV, pl6, and EGFR. The hrHPV, p16, and EGFR status of the tumors was correlated with CTP parameters (MTT, BV, BF, CP) by using the Wilcoxon evaluation and Fischer exact test.

RESULTS: There were significantly lower $C P$ values in pretreatment tumors overexpressing EGFR $(P=.04)$. CP values $\leq 17.23$ were significantly correlated with EGFR overexpression $(P=.015)$. A trend toward higher $C P$ values was present in hrHPV-positive and pl6overexpressing pretreatment tumors $(P=.14)$.

CONCLUSIONS: A significant correlation exists between CTP measures and EGFR overexpression in head and neck squamous cell carcinomas, suggesting an association between certain imaging findings and molecular biomarkers. These results may be related to a tumor cell survival mechanism linking perfusion and biomarker expression.

ABBREVIATIONS: $\mathrm{BF}=$ blood flow; $\mathrm{BV}=$ blood volume; $\mathrm{CP}$ = capillary permeability; $\mathrm{EGFR}=$ epidermal growth factor receptor; HPV = human papillomavirus; hrHPV = high-risk human papillomavirus

$\mathrm{T}$ here is increasing evidence that head and neck squamous cell carcinomas behave differently depending on their high-risk human papillomavirus status and p16 and EGFR overexpression. hrHPVs are polymorphic small deoxyribonucleic acid viruses that can disrupt the cell cycle, induce cellular

Received January 30, 2012; accepted after revision August 7.

From the Departments of Radiology (N.L.H.), Pathology (J.B.M.), Otolaryngology (B.K., H.W., M.P., C.B., T.E.C.), Cancer Biology Graduate Program (H.W.), and Depart ments of Radiology, Otolaryngology, Radiation Oncology, and Oral Medicine (S.K.M.), University of Michigan, Ann Arbor, Michigan; and Biostatistics Core (E.L.), and the Head and Neck SPORE Team, University of Michigan Comprehensive Cancer Center, Ann Arbor, Michigan.

This work was supported by the William N. Hanafee Seed Grant Award of the American Society of Head and Neck Radiologists, and the National Institutes of Health: NIDCR 1 R01-DE019126; NCI Head and Neck SPORE grant (1 P50 CA97248); NIDCD Research Center Core grant (P30 DC05188).

Paper previously presented in part at: Annual Meeting of the American Society of Head and Neck Radiologists, September 7-11, 2011; Coronado, California.

Please address correspondence to Nickoleta L. Hoefling, MD, Department of Radiology, State University of New York at Stony Brook, HSC L4 Room 120, Stony Brook, NY 11794-8460; e-mail: nickoleta.hoefling@stonybrook.edu immortalization, and lead to malignant transformation. ${ }^{1}$ hrHPV positivity is associated with head and neck squamous cell carcinomas, especially in patients with no history of alcohol or tobacco abuse. Patients with hrHPV-positive head and neck squamous cell carcinoma tumors have improved overall and disease-specific survival compared with patients with hrHPV-negative tumors. ${ }^{2,3}$

p16INK4A is a tumor-suppressor gene that is frequently mutated, leading to production of an abnormal protein, or downregulated, leading to too few copies of a normal protein, in multiple cancer types, including head and neck squamous cell carcinomas associated with tobacco and alcohol use. ${ }^{4-7}$ p16 may also be overexpressed, leading to too many copies of a normal protein, which is an independent prognostic marker in hrHPV-

\footnotetext{
- Indicates open access to non-subscribers at www.ajnr.org

三 Indicates article with supplemental appendix and on-line tables

http://dx.doi.org/10.3174/ajnr.A3349
} 
positive oropharyngeal squamous cell carcinoma, with p16 overexpressing tumors having greater 5-year survival, especially for tumor stages III/IV. ${ }^{8}$ Epidermal growth factor receptor is a transmembrane tyrosine kinase receptor that stimulates epidermal cell growth and is frequently overexpressed in head and neck squamous cell carcinoma tumors. ${ }^{9,10}$ EGFR overexpression is a risk factor for local recurrence and disease-specific death. ${ }^{11}$

CTP has been shown to be beneficial in identifying locally advanced head and neck squamous cell carcinomas that will respond to induction chemotherapy or chemoradiation versus those that will not respond. A prior study has suggested that tumors with higher blood flow and blood volume on pretreatment CTP examinations have a better response to nonsurgical organ-preservation therapy than tumors with lower initial $\mathrm{BV}$ and $\mathrm{BF} .{ }^{12}$ A recent study demonstrated significant changes in tumor BF, BV, and permeability surface area product after radiation therapy was given. ${ }^{13}$

Our purpose was to investigate the relationships between the CTP parameters of BV, BF, capillary perfusion, and mean transit time and the predictive molecular biomarkers of hrHPV, p16, and EGFR in head and neck squamous cell carcinoma tumors. We chose to look at both initially presenting pretreatment tumors and tumors that had previously undergone therapy, because previous work has shown differences in CTP measures between them. ${ }^{13}$ Our goal was to further advance the concept of an imaging biomarker profile that can help predict tumor response to treatment.

\section{MATERIALS AND METHODS Patient Population}

This study was an institutional review board-approved, Health Insurance Portability and Accountability Act-compliant, retrospective review of 25 patients with head and neck squamous cell carcinoma tumors. All patients had previously signed informed consent. Our study group (On-line Table 1) consisted of 8 women and 17 men, 41-80 years of age (average age, 60 years), divided into 2 groups: those on initial presentation with no previous head and neck squamous cell carcinoma treatment $(n=18)$; and those who had undergone previous treatment, whose carcinomas had recurred and were being re-evaluated before palliative therapy $(n=7)$. The pretreatment and palliative groups had similar distributions of $\operatorname{sex}(P=.36$, Fisher exact test $)$ and age $(P=.71$, $t$ test). The primary tumor sites in the pretreatment group included the tongue base $(n=5)$, tonsil $(n=3)$, false cord $(n=2)$, epiglottis $(n=2)$, tongue $(n=1)$, buccal mucosa $(n=1)$, glottis $(n=1)$, vallecula $(n=1)$, nasopharynx $(n=1)$, and retromolar trigone $(n=1)$. Tumor sites in the palliative group included the tongue $(n=1)$, floor of mouth $(n=1)$, masticator space $(n=1)$, parotid gland $(n=1)$, peristomal metastases $(n=2)$, and neck nodal disease $(n=1)$. All palliative group tumor biopsy samples were obtained from the recurrent tumor sites. Previous treatment in the palliative group included chemoradiation $(n=4)$ and radiation therapy $(n=3)$. Inclusion and exclusion criteria for the pretreatment and palliative patient groups are provided in the On-line Appendix.

\section{HPV Testing and Immunohistochemistry}

Tissue slides were recovered from pathology archives, deparaffinized, rehydrated, and peroxidase-quenched (Dako Cytoma-
Table 1: CTP parameter mean $(95 \% \mathrm{Cl})$ overall in pretreatment and palliative groups combined

\begin{tabular}{lc}
\hline CTP Parameter & Mean (95\% Cl) \\
\hline BF & $103.7(73.6-133.7)$ \\
BV & $11.8(3.7-19.9)$ \\
CP & $27.4(19.4-35.4)$ \\
MTT & $7.6(6.4-8.8)$ \\
\hline Note:-
\end{tabular}

Note:- $\mathrm{Cl}$ indicates confidence interval.

Table 2: CTP parameter mean $(95 \% \mathrm{Cl})$ by marker status in pretreatment and palliative groups combined

\begin{tabular}{cccc}
\hline & Positive & Negative & $\boldsymbol{P V}$ Value $^{\mathbf{a}}$ \\
\hline HPV & $(n=5)$ & $(n=19)$ & \\
BF & $121.4(56.8-186.1)$ & $101.6(63.5-139.6)$ & .53 \\
BV & $8.2(5.1-11.4)$ & $13.2(2.4-24.0)$ & .97 \\
CP & $30.4(9.8-51.1)$ & $27.3(17.4-37.2)$ & .67 \\
MTT & $7.4(3.3-11.6)$ & $7.8(6.5-9.1)$ & .52 \\
EGFR & $n=15$ & $n=5$ & \\
BF & $108.7(60.5-156.9)$ & $105.4(42.5-168.2)$ & .66 \\
BV & $14.7(0.8-28.6)$ & $7.4(5.4-9.4)$ & .93 \\
CP & $27.1(16.7-37.6)$ & $37.3(5.4-69.2)$ & .79 \\
MTT & $7.0(5.4-8.5)$ & $7.8(3.9-11.7)$ & .22 \\
\hline
\end{tabular}

Note:- $\mathrm{Cl}$ indicates confidence interval.

a $P$ value derived from the Wilcoxon 2-sample test.

tion, Glostrup, Denmark). All slides were incubated in Antigen Retrieval Solution (Dako Cytomation) for 40 minutes in a $92^{\circ} \mathrm{C}$ water bath with a buffer change midway and allowed to cool to room temperature for 20 minutes. For all samples tested for EGFR, an additional antigen-retrieval step was performed with pepsin incubation for 10 minutes at $37^{\circ} \mathrm{C}$. Horse serum was used for blocking (30 minutes at room temperature). Primary antibodies to EGFR/31G7 (Invitrogen, Camirillo, California) were added and allowed to incubate overnight at $4^{\circ} \mathrm{C}$. All staining for p16INK4a was performed per protocol supplied by the kit (CINtec p16INK4a Histology Kit; mtm Laboratories, Westborough, Massachusetts). Slides were washed, and secondary antibodies linked to avidin/biotin peroxidase (ABC Kit; Vector Laboratories, Burlingame, California) were used to detect primary antibody binding. For p16 and EGFR scoring, we used 2 scales of 1-4 for proportion of tumor cells stained and intensity of tumor cell staining. For proportion of stained cells: one was $<5 \%$; two, 5\%-20\%; three, $21 \%-50 \%$; and four, $51 \%-100 \%$. Tumor staining intensity was scored as 1 equal to no staining; 2 , low intensity; 3 , moderate; and 4, high intensity. Scores of $4(51 \%-100 \%)$ for the percentage of positive cells, coupled with intensity scores of 3 or 4 (moderate or high intensity), were considered positive for p16 and EGFR overexpression. Of note, all cases that were considered positive for p16 and EGFR overexpression were diffusely and strongly positive. HPV testing was performed using HPV-PCR MassARRAY (Sequenom, San Diego, California), which detects and identifies 15 high risk HPV subtypes using multiplex competitive PCR and single base extension followed by matrix-assisted laser desorption/ionization-time of flight mass spectroscopy, as previously described. ${ }^{14}$ All of the HPV positive tumors in this set contained HPV 16; 1 sample contained HPV 16, 35 and 66. In situ hybridization was carried out in the pathology laboratory on all tumor sections undergoing hrHPV testing, by using the Inform HPVIII in situ hybridization assay (Ventana, Tucson, Arizona), which consists of a cocktail directed against $12 \mathrm{hrHPV}$ genotypes (HPV 16, 18, 31, 33, 35, 39, 45, 51, 52, 56, 58, and 66). For hrHPV 
scoring, tumors were classified as positive if any nuclear signal was present and negative when no nuclear signal was present.

\section{Imaging Studies}

All imaging studies were performed on a multidetector scanner (LightSpeed Ultra; GE Healthcare, Milwaukee, Wisconsin). A noncontrast CT scan was obtained through the known primary tumor site. The area of interest for measuring perfusion was centered on the largest area of gross anatomic abnormality identified on the noncontrast images. Four adjacent 5-mm sections were selected at the level of the tumor. Fifty milliliters of nonionic contrast (iodine concentration, $300 \mathrm{mg} / \mathrm{mL}$; iopromide, Ultravist 300; Bayer HealthCare, Wayne, New Jersey) was injected at 4 $\mathrm{mL} / \mathrm{s}$. At 5 seconds into the injection, a cine (continuous) acquisition was initiated with the following parameters: $120 \mathrm{kV}, 60 \mathrm{~mA}$, $4 \times 5 \mathrm{~mm}$ sections. A region of interest was placed in the internal carotid artery to generate the contrast-enhancement curve. A single observer (a fellowship-trained neuroradiologist with 15 years' experience in head and neck imaging) who was aware of the primary tumor site subsequently drew regions of interest (25$30 \mathrm{~mm}^{2}$ ) through the primary tumor. Streak and other artifacts were avoided. The perfusion data were postprocessed by a deconvolution-based method into maps that represented $\mathrm{CP}, \mathrm{BV}, \mathrm{BF}$, and MTT by using the commercially available Perfusion- 4 software package on an Advantage Windows workstation (GE Healthcare).

\section{Statistical Analysis}

The 2 subgroups of pretreatment and palliative patients were analyzed separately and together. Analysis for demographic differences between the pretreatment and palliative groups was performed with the Fisher exact test for sex and the $t$ test for age. Nonparametric Wilcoxon rank-based statistics were used for correlating the imaging biomarkers (BV, BF, MTT, CP) and the laboratory biomarkers (EGFR, hrHPV, p16). The nonparametric Wilcoxon test assigned relative ranks to each individual value; the ranks were then compared across discrete groups. The Wilcoxon rank-based test was also used to determine whether differences in the CTP parameters existed between the pretreatment and palliative groups. The Fischer exact test was used for determining which CP values were predictive of the EGFR status of tumors. SAS, Version 9.2 statistical software (SAS Institute, Cary, North Carolina) was used for all calculations.

\section{RESULTS}

All (18/18) of the pretreatment patients' tumor biopsy samples were tested for hrHPV, and 14/18 were tested for p16 and EGFR overexpression. All (7/7) of the palliative patients' tumor specimens were tested for hrHPV and p16 overexpression, and 6/7 were tested for EGFR overexpression (On-line Table 1). The 4/18 pretreatment samples that were not tested for p16 and EGFR and the $1 / 7$ palliative samples that was not tested for EGFR were not tested due to insufficient sample quantity or quality. The CTP parameters of BF, BV, MTT, and CP (On-line Table 2) were calculated for all 25 patients.
Table 3: CTP parameter mean $(95 \% \mathrm{Cl})$ overall in the pretreatment group

\begin{tabular}{lc}
\hline CTP Parameter & Mean $(95 \% \mathrm{Cl})$ \\
\hline BF & $124.2(87.4,160.9)$ \\
BV & $8.4(6.1,10.7)$ \\
CP & $19.9(15.8,24.0)$ \\
MTT & $7.1(5.7,8.5)$ \\
\hline
\end{tabular}

Note:- Cl indicates confidence interval.

Table 4: CTP parameter mean $(95 \% \mathrm{Cl})$ by marker status in the pretreatment group

\begin{tabular}{cccc}
\hline CTP Parameter & Positive & Negative & $\boldsymbol{P V a l u e}^{\mathbf{a}}$ \\
\hline HPV & $n=4$ & $n=13$ & \\
BF & $134.5(55.2-213.8)$ & $126.3(77.0-175.7)$ & .69 \\
BV & $8.2(3.5,12.8)$ & $8.9(5.9-12.0)$ & .95 \\
CP & $23.5(12.0-35.0)$ & $19.4(14.1-24.6)$ & .14 \\
MTT & $7.3(1.2-13.3)$ & $7.2(5.6-8.8)$ & .95 \\
EGFR & $n=10$ & $n=4$ & \\
BF & $137.1(70.2-204.0)$ & $108.8(16.9-200.7)$ & .72 \\
BV & $8.7(4.4-13.1)$ & $7.0(4.4-9.7)$ & .94 \\
CP & $16.2(11.8-20.7)$ & $26.7(8.9-44.4)$ & .04 \\
MTT & $5.8(4.3-7.3)$ & $7.9(2.1-13.7)$ & .44 \\
\hline
\end{tabular}

Note:- $\mathrm{Cl}$ indicates confidence interval.

a $P$ value derived from the Wilcoxon 2-sample test.

\section{Correlation between hrHPV Positivity and p16 Overexpression}

All hrHPV-positive samples that were tested for p16 showed p16 overexpression $(\kappa=1.0)$.

\section{Combined Analysis of All Patients}

No significant correlations were found between any of the laboratory biomarkers (p16, hrHPV, EGFR) and the CT perfusion biomarkers (BV, BF, MTT, CP) when the entire patient group $(n=25)$ was analyzed together (Tables 1 and 2).

\section{Association of CTP Parameters and Laboratory Biomarkers in the Pretreatment Group}

Analysis of the pretreatment patient group $(n=18)$ (Tables 3 and 4 ) alone demonstrates significantly decreased $\mathrm{CP}$ values in EGFR-overexpressing tumors $(P=.04)$ (Fig 1$)$. Eighty percent of EGFR-overexpressing tumors had CP values $\leq 17.23(P=.015)$. There was a trend toward increased CP values in hrHPV-positive and p16-overexpressing tumors $(P=.14)$ (Fig 2).

\section{Association of CTP Parameters and Laboratory Biomarkers in Palliative Patients}

No significant correlations were found between any of the laboratory biomarkers (p16 overexpression, EGFR overexpression, and hrHPV positivity) and the CTP parameters of BF, BV, CP, and MTT for the palliative patient group $(n=7)$.

\section{Differences in CTP Parameters between Pretreatment and Palliative Patients}

Pretreatment patients had significantly lower CP (Table 5) values than patients in the palliative group $(P=.01)$ (Fig 3$)$, despite the fact that the lowest $\mathrm{CP}$ value, 1.13, was reported in a palliative patient. There was significantly higher BF in the pretreatment group compared with the palliative patient group $(P=.01)$ (Fig 4). 


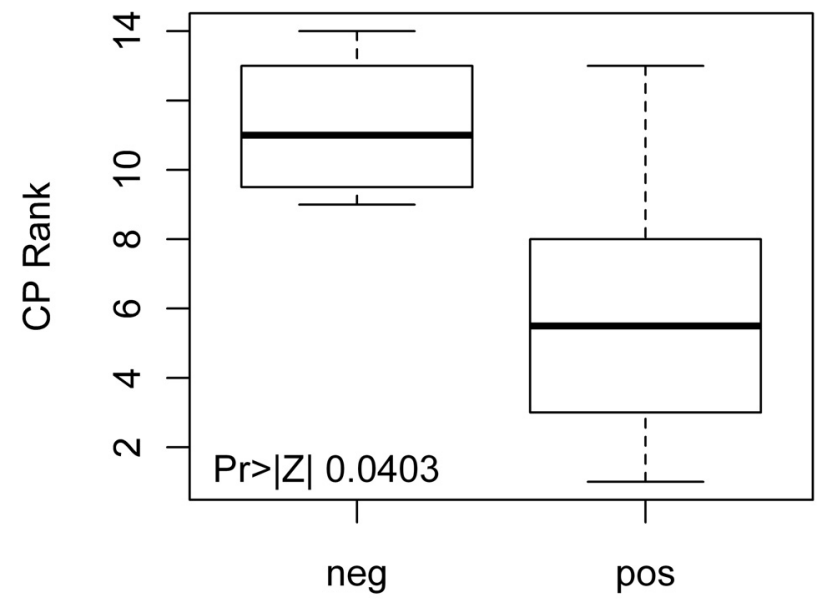

EGFR

FIG 1. Distribution of Wilcoxon scores for CP in pretreatment patients. We see significantly lower CP in pretreatment patient tumors that overexpress EGFR.

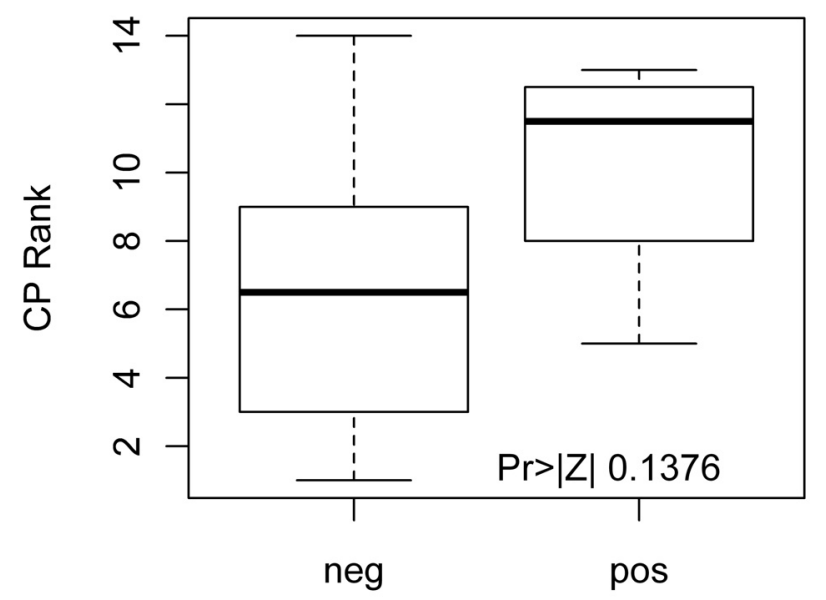

p16 and HPV

FIG 2. Distribution of Wilcoxon scores for $C P$ in pretreatment patients. There is a trend toward higher $\mathrm{CP}$ in tumors that overexpress pl6 and are HPV-positive.

Table 5: CTP parameter mean $(95 \% \mathrm{CI})$ by pretreatment-versuspalliative status

\begin{tabular}{lclc}
\hline & Pretreatment $(\boldsymbol{n}=18)$ & Palliative $(\boldsymbol{n}=7)$ & $\boldsymbol{P}$ Value \\
\hline BF & $124.2(87.4-160.9)$ & $50.9(19.1-82.7)$ & .01 \\
BV & $8.4(6.1-10.7)$ & $20.4(-13.6-54.5)$ & .86 \\
CP & $19.9(15.8-24.0)$ & $46.5(21.8-71.2)$ & .01 \\
MTT & $7.1(5.7-8.5)$ & $9.0(6.8-11.3)$ & .15 \\
\hline
\end{tabular}

a $P$ value derived from the Wilcoxon 2-sample test.

\section{DISCUSSION}

Our study demonstrates that the CT perfusion parameter of capillary permeability is significantly correlated with overexpression of EGFR in pretreatment patients, advancing the growing concept that noninvasively acquired imaging biomarkers may be predictive of molecular biomarkers. A previous study by Ash et $\mathrm{al}^{15}$ identified a correlation between the CTP parameters of blood flow and blood volume and microvascular density. Our study

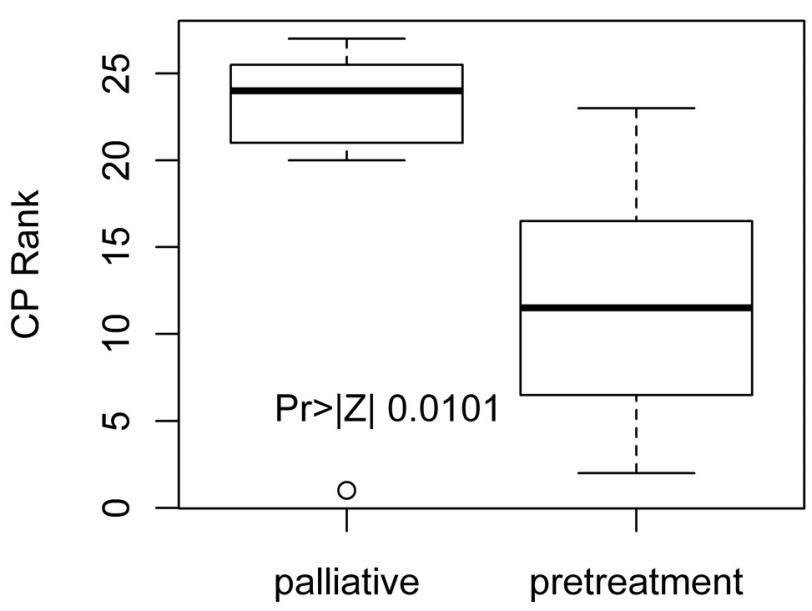

\section{Palliative}

FIG 3. Distribution of Wilcoxon scores for $C P$ in pretreatment versus palliative patients. Pretreatment patients have significantly lower CP scores versus palliative patients.

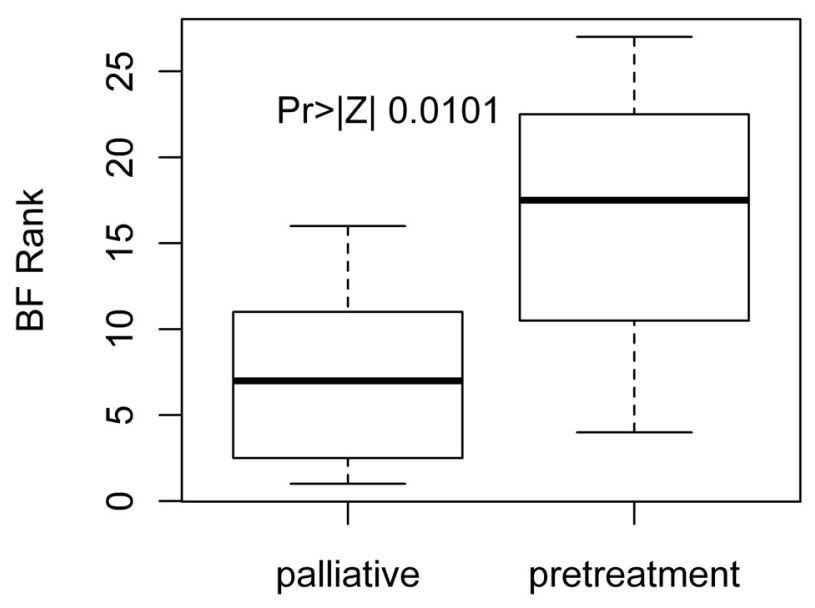

\section{Palliative}

FIG 4. Distribution of Wilcoxon scores for BF in pretreatment versus palliative patients. We see that pretreatment patients have significantly higher $\mathrm{BF}$ versus palliative patients.

adds to this work, suggesting that CTP may predict both histologic microvasculature parameters and tumoral laboratory biomarkers.

These results also support the growing use of imaging as a noninvasive method for risk stratification to assess which patients with head and neck squamous carcinoma are best treated with nonsurgical organ-preservation therapy. BF measurements have been found to differentiate recurrent tumor from posttreatment changes, demonstrating the ability of CTP to detect tissue changes present after chemoradiation. ${ }^{16}$ It has also been shown that while baseline blood volume predicts short-term response, follow-up CTP can also suggest which patients are continuing to respond during the course of chemoradiation. ${ }^{13}$

Our statistical analysis revealed unexpected findings that further increased our belief that imaging biomarkers can predict molecular biomarkers. The correlation between CP and EGFR was only present 
in previously untreated patients $(n=18)$. When we included the 7 patients who had failed treatment, there was no significant correlation between CP and EGFR overexpression, suggesting that the molecular composition of these recurrent tumors differs from that in the pretreatment group. The concept that there are intrinsic differences in untreated tumors versus recurrent tumors is supported by our other findings that the pretreatment patient cohort had significantly lower $\mathrm{CP}$ values $(P=.01)$ and higher $\mathrm{BF}(P=.01)$ than patients in the palliative group (Figs 3 and 4 ).

The exact cause of this difference between untreated and recurrent tumors is unclear. Previous work has shown significant changes in tumor BF, BV, and permeability surface area product after radiation therapy was given. ${ }^{13}$ It is possible that treatment could alter the cell-surface expression of EGFR. Another hypothesis is based on tumor heterogeneity. Some tumors may not have had a homogeneous population, so that successful treatment was limited to those cells without EGFR overexpression. EGFR overexpressing tumors have been shown to be more likely to recur and to be less responsive to chemoradiation. ${ }^{11,17}$ Tumor overexpression of EGFR has been associated with poorer response to induction chemotherapy and decreased overall survival and disease-specific survival. ${ }^{18}$ Our results fit with previous work that has shown that a lower CT perfusion parameter of permeability surface area product value correlates with locoregional recurrence. ${ }^{19}$ Our study was not designed, however, to perform CTP and tissue sampling pre- and posttreatment. Thus, we cannot confirm whether there was a change in EGFR expression in recurrent tumors compared with pretreatment expression. The molecular basis of the correlation between lower CP values and EGFR overexpression in pretreatment tumors is unknown and should be the subject of future investigations.

There are several limitations to our study. First, our data were reviewed in a retrospective manner, and the patient population was relatively small and from a single institution. Second, some of the original tumor biopsy samples did not have enough tissue remaining to test for all 3 laboratory biomarkers. While this may introduce bias in the results, each laboratory biomarker was independently correlated with each CTP parameter to limit this bias. Furthermore, each CTP study was evaluated by a single observer. Other limitations include lack of generalizability of the processing software and lack of an internal reference for normalization. Larger studies performed prospectively and at multiple institutions would be necessary to confirm our findings.

\section{CONCLUSIONS}

Our exploratory study found significant correlations between the CTP measure of CP and the EGFR status of tumors. The results of our investigation suggest that CT perfusion imaging biomarkers can be used to predict important molecular biomarkers. Our results further support the concept of creating a molecular imaging profile that can be used to assess risk stratification. This initial analysis may serve as the foundation for future large-scale investigations that further seek to determine whether there are other direct correlations between imaging and molecular biomarkers.

Disclosures: Suresh K. Mukherji-UNRELATED: Consultancy: Philips Medical Systems, Comments: not related to work done in the manuscript.

\section{REFERENCES}

1. Chung C, Gillison M. Human papillomavirus in head and neck cancer: its role in pathogenesis and clinical implications. Clin Cancer Res 2009; 15:6758-62

2. D’Souza G, Kreimer A, Viscidi R, et al. Case-control study of human papillomavirus and oropharyngeal cancer. N Engl J Med 2007;356: $1944-56$

3. Gillison ML, Koch WM, Capone RB, et al. Evidence for a causal association between human papillomavirus and a subset of head and neck cancers. J Natl Cancer Inst 2000;92:709-20

4. Yuen PW, Man M, Lam KY, et al. Clinicopathological significance of p16 gene expression in the surgical treatment of head and neck squamous cell carcinomas. J Clin Pathol 2002;55:58-60

5. Nobori T, Miura K, Wu DJ, et al. Deletions of the cyclin-dependent kinase-4 inhibitor gene in multiple human cancers. Nature 1994; 368:753-56

6. Kamb A, Gruis NA, Weaver-Feldhaus J, et al. A cell cycle regulator potentially involved in genesis of many tumor types. Science 1994;264:436-40

7. Wong TS, Man MW, Lam AK, et al. The study of p16 and p15 gene methylation in head and neck squamous cell carcinoma and their quantitative evaluation in plasma by real-time PCR. Eur J Cancer 2003;39:1881-87

8. Fischer CA, Kampmann M, Zlobec I, et al. p16 expression in oropharyngeal cancer: its impact on staging and prognosis compared with the conventional clinical staging parameters. Ann Oncol 2010;21: 1961-66

9. Santini J, Formento JL, Francoual M, et al. Characterization, quantification, and potential clinical value of the epidermal growth factor receptor in head and neck squamous cell carcinomas. Head Neck 1991;13:132-39

10. Kalyankrishna S, Grandis J. Epidermal growth factor receptor biology in head and neck cancer. J Clin Oncol 2006;24:2666-72

11. Fei J, Hong A, Dobbins T, et al. Prognostic significance of vascular endothelial growth factor in squamous cell carcinomas of the tonsil in relation to human papillomavirus status and epidermal growth factor receptor. Ann Surg Oncol 2009;16:2908-17

12. Zima A, Carlos R, Gandhi D, et al. Can pretreatment CT perfusion predict response of advanced squamous cell carcinoma of the upper aerodigestive tract treated with induction chemotherapy? AJNR Am J Neuroradiol 2007;28:328-34

13. Surlan-Popovic K, Bisdas S, Rumboldt Z, et al. Changes in perfusion CT of advanced squamous cell carcinoma of the head and neck treated during the course of concomitant chemoradiotherapy. AJNR Am J Neuroradiol 2010;31:570-75

14. Tang AL, Hauff SJ, Owen JH, et al. UM-SCC-104: a new human papillomavirus-16 positive-cancer stem cell-containing head and neck squamous cell carcinoma cell line. Head Neck 2012;34:1480-91

15. Ash L, Teknos T, Gandhi D, et al. Head and neck squamous cell carcinoma: CT perfusion can help noninvasively predict intratumoral microvessel density. Radiology 2009;251:422-28

16. Bisdas S, Baghi M, Smolarz A, et al. Quantitative measurements of perfusion and permeability of oropharyngeal and oral cavity cancer, recurrent disease, and associated lymph nodes using first-pass contrast-enhanced computed tomography studies. Invest Radiol 2007;42:172-79

17. Kumar B, Cordell K, Lee J, et al. EGFR, p16, HPV titer, Bcl-xL and p53, sex, and smoking as indicators of response to therapy and survival in oropharyngeal cancer. J Clin Oncol 2008;26: 3128-37

18. Pivot X, Magn N, Guardiola E, et al. Prognostic impact of the epidermal growth factor receptor levels for patients with larynx and hypopharynx cancer. Oral Oncol 2005;41:320-27

19. Bisdas S, Nguyen S, Anand S, et al. Outcome prediction after surgery and chemoradiation of squamous cell carcinoma in the oral cavity, oropharynx, and hypopharynx: use of baseline perfusion CT microcirculatory parameters vs. tumor volume. Int J Radiat Oncol Biol Phys 2009;73:1313-18 